\title{
Inhibition of vection by red
}

\author{
Takeharu Seno, Shoji Sunaga, and Hiroyuki Ito \\ Kyushu University, Fukuoka, Japan
}

\begin{abstract}
We investigated the effects of colors on vection induction. Expanding optical flows during one's forward self-motion were simulated by moving dots. The dots and the background were painted in equiluminant red and green. Experiments 1 and 2 showed that vection was weaker when the background was red than when the background was green. In addition, Experiment 3 showed that vection was weaker when the moving dots were red than when the dots were green. Experiment 4 demonstrated that red dots on a red background induced very weak vection, as compared with green dots on a green background. In Experiments 5 and 6, we showed that the present results could not be explained by a luminance artifact. Furthermore, Experiment 7 showed that a moving red grating induced weaker vection than did a green one. We concluded that a red visual stimulus inhibits vection.
\end{abstract}

When one is exposed to a visual motion field that simulates the retinal optical flow generated by one's own movement, one often perceives a subjective movement of one's body. This phenomenon is called vection (Fischer \& Kornmuller, 1930). Several stimulus attributes are known to affect the subjective strength or direction of vection, such as depth (e.g., Ito \& Shibata, 2005), visual area (e.g., Brandt, Dichgans, \& Koenig, 1973), motion direction (e.g., Seno \& Sato, 2009), and attention (e.g., Kitazaki \& Sato, 2003). To date, color is one of the stimulus attributes in vection induction that has rarely been tested. In almost all previous studies, achromatic stimuli were employed to induce vection - that is, white dots on a black background (e.g., Bubka, Bonato, \& Palmisano, 2008) or achromatic gratings (e.g., Brandt et al., 1973). No published study has ever investigated the effect of color on vection, except for Bonato and Bubka (2006). In the present study, we focused on the effect of color in vection, especially on the effect of red.

Red has been known as a color that has certain inhibiting effects on visual performance. Psychophysical studies have revealed that the magnitude of metacontrast masking decreases on the presentation of a red background (Bedwell, Brown, \& Miller, 2003; Breitmeyer \& Williams, 1990; Edwards, Badcock, \& Nishida, 1996; Pammer \& Lovegrove, 2001). Global precedence effects in hierarchically organized stimuli were weaker with a red background than with an equiluminant green background (Michimata, Okubo, \& Mugishima, 1999). Under normal luminance contrast conditions, red light impairs reading performance (Chase, Ashourzadeh, Kelly, Monfette, \& Kinsey, 2003). For motion perception, Chapman, Hoag, and Giaschi (2004) demonstrated that adaptation to a red visual field results in increased motion coherence thresholds. These results have been interpreted as showing that red visual fields inhibit the magnocellular pathway - that is, the motion pathway (this interpretation is discussed later). The optic flows that induce vection are a type of coherent motion. Thus, it is plausible that a red background has an effect on vection strength when it is employed in a vection stimulus.

In this study, we measured vection strength by using a red background and red dots. If the motion pathway is inhibited by a large red field, such a field should affect vection, as well as coherent motion, as was observed by Chapman et al. (2004). We used flicker photometry to generate isoluminant red and green visual fields. These colors were used as backgrounds for the vection stimuli as wellthat is, the optic flows. The luminance contrast between the dots and the background was constant between the two (red and green). Under this condition, we examined whether the inhibition of vection by red exists or not.

In Experiment 1, we used optic flows with bright red dots on a dark green background and also with bright green dots on a dark red background. We hypothesized that if a red background inhibits the motion pathway, vection should be weaker in the red background condition than in the green background condition. Although the color combinations were opposite in the two conditions, the luminance contrast between the dots and the background was unchanged. Thus, if there is a difference in vection strength between the two, it should originate in the color, not the luminance. In Experiment 2, we used optic flows with white dots on red and green backgrounds and allowed subjects to adapt to the background for $20 \mathrm{sec}$. In Experiment 3, we used optic flows with red and green dots on a black background. In Experiment 4, we used optic flows with red dots on a red background or green dots on a green background. Experiment 5 was conducted to show that vection inhibition by red dots was not mediated by luminance-setting artifacts. In Experiment 6, we examined the possible amount of background luminancesetting artifacts included in the results of the former ex-

T. Seno, seno@design.kyushu-u.ac.jp 
periments and provided the control conditions by using achromatic optic flow. Finally, in Experiment 7, we further tested the effect of color with red and green monochromatic gratings.

\section{GENERAL METHOD}

\section{Apparatus}

Stimulus images (pixel resolution, 1,024 $\times 768$; refresh rate, $75 \mathrm{~Hz}$ ) were generated and controlled by a computer (Apple MB543J/A). They were projected onto a translucent screen by a rear 3-CRT projector (DRAPAR; Electrohome Electronics). The experiments were conducted in a darkened room.

\section{Stimuli}

In Experiments 1-6, the stimuli were expanding optic flow patterns that subtended a visual angle of $75^{\circ}$ (horizontal) $\times 60^{\circ}$ (vertical) at a viewing distance of $90 \mathrm{~cm}$. The screen size was $118 \mathrm{~cm}$ (horizontal) $\times 85 \mathrm{~cm}$ (vertical). The optic flows were generated using the OpenGL software. We positioned 16,000 dots at random in a simulated cube (length, $20 \mathrm{~m}$ ), and the viewpoint was moved to simulate forward self-motion $(6 \mathrm{~m} / \mathrm{sec})$. The start and the end points of the cube were connected, thus allowing endless optic flows to be generated. Approximately 1,240 dots were presented in each frame, and each dot subtended a visual angle of $0.03^{\circ}-0.05^{\circ}$. The density of the dots was $0.1 \%$ to $0.2 \%$ of the whole screen, and density was constant over the whole screen; 0.3 dots existed per square degree. The velocity of the dots ranged from 0.1 to $15 \mathrm{deg} / \mathrm{sec}$. It was possible for us to set the flow speed faster to increase vection strength. However, if we had used faster stimuli, vection could easily have been saturated. This would result in erasing any difference in vection strength between the experimental conditions. The sizes of the dots remained unchanged even when the simulated distance changed. Moreover, the dots did not form a density gradient. To achieve the absence of a density gradient in a 2-D visual stimulus, we manipulated the density of the dots in the 3-D simulated space. The density gradient in the 3-D space canceled out the dot density gradient in the 2-D space. In the present experiments, a space within a $20-\mathrm{m}$ depth was simulated. More dots appeared at depth positions closer to the simulated viewpoint. The dots disappeared when they reached the depth position of the screen. Therefore, the dots appeared suddenly from every part of the visual field and disappeared after each dot lifetime. As a result, there were no depth cues, except for motion parallax. In Experiment 7, we used grating stimuli, as noted later.

The colors used in the dots and the background were red, green, and white. The CIE $1931 x, y$ chromaticity coordinates of the red, the green, and the white were $(.645, .350),(.350, .577)$, and $(.224, .229)$, respectively. The CIE $1931 x, y$ chromaticity coordinates of the red and the green at a screen position corresponding to a $30^{\circ}$ peripheral visual field were $(.625, .362)$ and $(.338, .569)$, respectively. Thus, a constant chromaticity was obtained over the entire visual field. The colors and luminances of the dots and the background of the stimulus were set according to the purpose of each experiment.

\section{Procedure}

The optic flow pattern was presented for $100 \mathrm{sec}$, during which we measured latency, duration, and subjective strength of vection. On each trial, the subject's task was to keep pressing a button throughout the period during which they perceived vection. Latency was defined as the time interval between the onset of the stimulus presentation and the time at which the subject first pressed the button to indicate that he or she perceived vection, and duration was calculated as the total time for which the button was pressed. After stimulus presentation, we asked the subject to report the subjective strength of vection by using the magnitude estimation method. The estimated values ranged from 0 (no vection) to 100 (very strong vection).
We conducted five successive trials for each condition in Experiments 1-5 and three and four trials in Experiments 6 and 7. To enhance the adaptation effect, four or five successive trials were conducted under one condition, except for Experiment 6 . The subjects were allowed to rest between the trials for each condition. The order of the red and green conditions was counterbalanced between the 12 subjects. For example, 6 of the 12 subjects performed five of the red background trials successively, followed by five of the green background trials, and the other 6 subjects performed the trials in the opposite order. For ethical reasons (to avoid motion sickness), we permitted the subjects to freely determine the length and timing of their rest periods.

The instructions read, "Please press the corresponding button while perceiving self-motion. If such a decision becomes difficult, please release the buttons." We did not give them any suggestions that might lead to any cognitive bias with respect to the color effect hypothesis, because vection can be modulated by such instructions (e.g., Lepecq, Giannopul, \& Baudonniere, 1995; Palmisano \& Chan, 2004). The subjects practiced pressing the buttons before starting the experimental trials.

\section{Subjects}

Twelve naive subjects participated in Experiments 1-7. They were either graduate or undergraduate students (20-27 years of age, 8 males and 4 females). All of them had normal color vision that we assessed by the Fransworth-Munsell 100-hue test, and they had not experienced any disease of the vestibular system. All of them had previously participated in vection experiments and in demos in psychology lectures before participating in this experiment. Informed consent was obtained from each subject.

\section{EXPERIMENT 1}

\section{Method}

In Experiment 1, there were two color conditions. In one of them, the color of the dots was green, whereas the color of the background was red (the $\mathrm{G} / \mathrm{R}$ condition). The other condition was the converse of the $\mathrm{G} / \mathrm{R}$ condition - that is, red dots on a green background (the $\mathrm{R} / \mathrm{G}$ condition).

The luminances of red and green used for the dots were subjectively equal, as were the luminances of red and green for the background. The individual isoluminance between red and green was measured in flicker photometry $(20 \mathrm{~Hz})$ on the whole screen. In flicker photometry, the luminance of red was fixed at $1.0 \mathrm{~cd} / \mathrm{m}^{2}$ for the background and $5.6 \mathrm{~cd} / \mathrm{m}^{2}$ for the dot. The subjects could adjust the luminance of green. The luminances of green that equated to red ranged from 1.0 to $1.2 \mathrm{~cd} / \mathrm{m}^{2}$ for the background and from 5.5 to $5.7 \mathrm{~cd} / \mathrm{m}^{2}$ for the dot.

The luminances of the green and red backgrounds on the $30^{\circ}$ peripheral screen were around 0.5 and $0.3 \mathrm{~cd} / \mathrm{m}^{2}$, respectively, when they were $1.0 \mathrm{~cd} / \mathrm{m}^{2}$ at the center of the screen. The luminances of green and red dots on the $30^{\circ}$ peripheral screen were 0.9 and $1.0 \mathrm{~cd} / \mathrm{m}^{2}$, respectively, when they were $5.6 \mathrm{~cd} / \mathrm{m}^{2}$ at the center of the screen. The luminances decreased toward peripheral screen positions. However, the tendencies of the decreases were similar between the red and green luminances.

A low luminance contrast between the dots and the background results in a subjective disappearance of the peripheral dots, and such stimuli cannot induce vection. Therefore, in order to maintain a high luminance contrast between the dots and the background, we used a darker background than that used previously (Chapman et al., 2004).

\section{Results and Discussion}

We found that the latency became longer, the duration became shorter, and the magnitude values became smaller in the $\mathrm{G} / \mathrm{R}$ condition than in the $\mathrm{R} / \mathrm{G}$ condition (Figures 1 


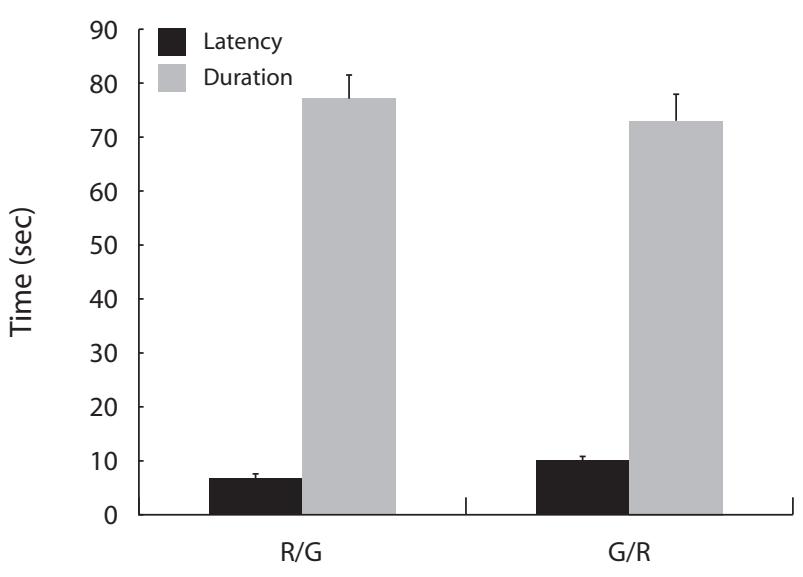

Figure 1. Average latency and duration of vection for the 12 subjects in each condition in Experiment 1. The error bars indicate standard errors. $R / G$, red dots on green background; $G / R$, green dots on red background.

and 2). The $t$ tests revealed significant differences between the two conditions [latency, $t(11)=2.50, p<.05$; duration, $t(11)=2.19, p=.05$; magnitude, $t(11)=2.71, p<$ $.05]$. These results indicate that vection was weaker in the $\mathrm{G} / \mathrm{R}$ condition. There was no difference in effective luminance between the two conditions. Therefore, these results were caused by the color difference and point toward inhibition by a red background. It could be argued that the green dots, and not the red background, could have inhibited vection. Therefore, in Experiments 2 and 3, we tested the two factors - that is, dot color and background color-separately.

\section{EXPERIMENT 2}

\section{Method}

There were two factors of dot and background colors included in the vection stimulus in Experiment 1. In Experiment 2, we investigated the effect of the background color on vection.

The color of the dots in the optic flows was changed to a higher luminance white of $36.7 \mathrm{~cd} / \mathrm{m}^{2}$. The colors and luminances of the two backgrounds were the same as those in Experiment 1. Thus, in Experiment 2, the optic flow patterns were presented under a whitedots-on-a-red-background (W/R) condition and a white-dots-on-agreen-background $(\mathrm{W} / \mathrm{G})$ condition.

Moreover, to enhance the effect of the background color on motion pathway, as Chapman et al. (2004) did, a 20-sec period of adaptation to the colored background preceded the presentation of the expanding optic flow pattern on each trial.

\section{Results and Discussion}

Figures 3 and 4 show the average latency, duration, and estimated magnitude of vection under the two (W/R and $\mathrm{W} / \mathrm{G}$ ) conditions. The average latency was approximately $11 \mathrm{sec}$ for the W/R condition-considerably longer than the latencies for normal forward vection (e.g., 4-7 sec for the optical flow stimulus; Bubka et al., 2008). In the W/R condition, the average duration was approximately $70 \mathrm{sec}$, and the average magnitude was about 40 . In the $\mathrm{W} / \mathrm{G}$ condition, the latency was approximately $6 \mathrm{sec}$, the duration was $85 \mathrm{sec}$, and the average magnitude was approximately 60 . The $t$ tests revealed that the background color had a significant effect on the duration $[t(11)=2.59, p<.05]$, on the latency $[t(11)=2.58, p<.05]$, and on the magnitude of vection $[t(11)=3.53, p<.05]$.

The indices of vection strength indicated that the maintenance and the subjective strength of vection were weaker in the $\mathrm{W} / \mathrm{R}$ condition than in the $\mathrm{W} / \mathrm{G}$ condition. Thus, these results can be interpreted as inhibition of vection by a red background. The difference between the $\mathrm{W} / \mathrm{R}$ and the $\mathrm{W} / \mathrm{G}$ conditions was more exaggerated than the difference between the $\mathrm{G} / \mathrm{R}$ and the $\mathrm{R} / \mathrm{G}$ conditions.

\section{EXPERIMENT 3}

\section{Method}

In Experiment 3, we investigated solely the effect of dot color on vection. In contrast to Experiment 2, the luminances and colors of the backgrounds used in this experiment were identical in both (green dot and red dot) conditions - that is, black of $0 \mathrm{~cd} / \mathrm{m}^{2}$. Thus, the optic flow patterns used here constituted the red-dots-ona-black-background (R/B) condition and the green-dots-on-a-blackbackground $(\mathrm{G} / \mathrm{B})$ condition. The luminances of the red and green dots were the same as those in Experiment 1-that is, they were subjectively isoluminant.

\section{Results and Discussion}

We found that the latency became longer, the duration became shorter, and the magnitude values became smaller in the $\mathrm{R} / \mathrm{B}$ condition than in the $\mathrm{G} / \mathrm{B}$ condition (Figures 5 and 6). The $t$ tests revealed significant differences between the two conditions [latency, $t(11)=2.65, p<.05$; duration, $t(11)=3.53, p<.01$; magnitude, $t(11)=2.75, p<$ $.05]$. These results indicate that vection was weaker in the $\mathrm{R} / \mathrm{B}$ condition than in the $\mathrm{G} / \mathrm{B}$ condition. Surprisingly, the inhibitory effect of red on vection appears even with the dot color and is not restricted to the background color. It appears that red has an inhibitory effect on vection irrespective of its role in the stimulus.

In Experiment 2, we obtained the inhibitory effect from the red background, and in Experiment 3, we obtained the

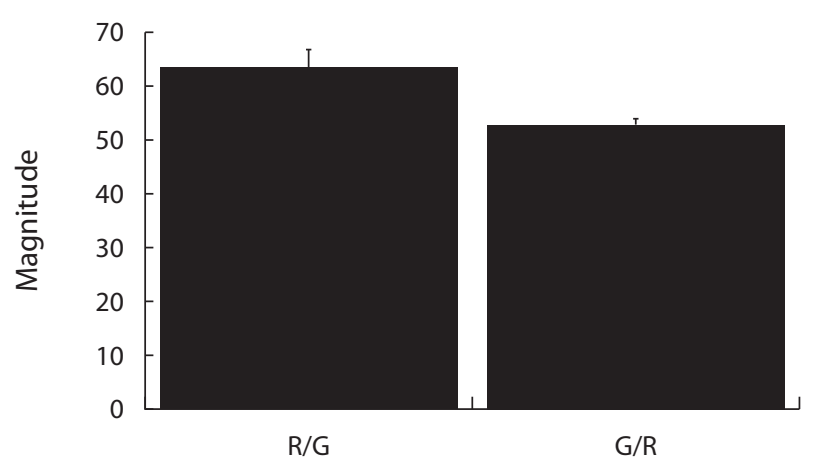

Figure 2. Average magnitude of vection for the 12 subjects in each condition in Experiment 1. The abscissas indicate the colors of the dots and the backgrounds: $R / G$, red dots on green background; $G / R$, green dots on red background. The error bars indicate standard errors. 


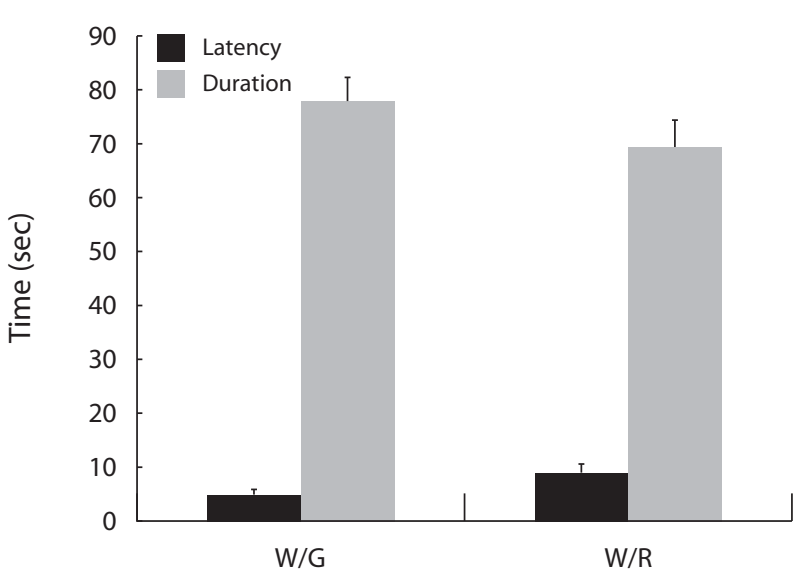

Figure 3. Average latency and duration of vection for the 12 subjects in each condition in Experiment 2. W/G, white dots on green background; $W / R$, white dots on red background. The error bars indicate standard errors.

same effect from the red dots. On the basis of these results, vection in the $\mathrm{R} / \mathrm{G}$ condition in Experiment 1 should also have been inhibited because of the red dots. In addition, the results of Experiment 1 showed that vection was more inhibited by the G/R condition. Thus, we conclude that the inhibition by red is more effective when the background is red than when the dots are, although it can be affected by the dots' size, density, and total area. The smaller difference between the $\mathrm{G} / \mathrm{R}$ and the $\mathrm{R} / \mathrm{G}$ conditions may indicate that the red dots and red background inhibited vection and canceled out each other in the $G / R$ and $R / G$ conditions. The results obtained in Experiment 3 indicate that vection under the $\mathrm{R} / \mathrm{B}$ condition was more attenuated than the vections obtained in Experiment 2. This may have been related to the visibility of the dots. The luminance difference between the dots and the background in Experiment 3 was much smaller, as compared with that of the stimuli in Experiment 2.

From a skeptical point of view, the results in Experiment 3 could be construed as an artifact of equiluminance settings. When the effective luminance contrast of red is lower than that of green, vection should be weaker in the $\mathrm{R} / \mathrm{B}$ condition. Thus, we examined this possibility later in Experiment 5.

\section{EXPERIMENT 4}

The results from Experiments 1-3 indicate that red inhibits vection. The dots and the background, separately, demonstrated the inhibitory effect of red on vection. However, these conditions always included a color difference between the dots and the background; that is, the moving dots were defined by differences in luminance and in color. If some interaction in motion processing between luminance and color motions produced the vection inhibition by red, no inhibition would arise when the dots and the background were in the same color. We compared conditions in which red dots were on a red background (the $\mathrm{R} / \mathrm{R}$ condition) and in which green dots were on a green background (the $\mathrm{G} / \mathrm{G}$ condition). In these two conditions, the luminance contrast between the dots and the background was identical, the only difference being the color of the stimuli.

\section{Method}

Red and green were used for both the dots and the background of the optic flows. The red and green for the dots and the background were set to the luminances that were measured by flicker photometry, as in Experiment 1.

\section{Results and Discussion}

Relatively weak vection was obtained in the $\mathrm{R} / \mathrm{R}$ condition, whereas strong vection was obtained in the G/G condition. The latency became longer, the duration became shorter, and the magnitude values became smaller in the $\mathrm{R} / \mathrm{R}$ condition than in the $\mathrm{G} / \mathrm{G}$ condition (Figures 7 and 8). The $t$ tests revealed significant differences between the two conditions [latency, $t(11)=2.45, p<.05$; duration, $t(11)=2.70, p<.05$; magnitude, $t(11)=5.80, p<.01]$. These results indicate that vection was weaker in the $R / R$ condition than in the $\mathrm{G} / \mathrm{G}$ condition. The differences in latency and duration were the second largest in Experiments $1-4$. The difference in the estimated magnitude, especially, was greater here than in all the former experiments. Vection strength measured with the magnitude estimation was found to be nearly half in the $\mathrm{R} / \mathrm{R}$ condition, as compared with the $\mathrm{G} / \mathrm{G}$ condition.

The combination of red dots on a red background was very effective in the inhibition of vection, although the luminance contrast was identical in the two conditions.

\section{EXPERIMENT 5}

As was mentioned above, an artifact in luminance settings - that is, the residual effective luminance difference between the red dots and the green dots - could result in weaker vection in the $\mathrm{R} / \mathrm{B}$ condition in Experiment 3 or in the $\mathrm{R} / \mathrm{R}$ condition in Experiment 4 . It is possible that the lower effective luminance of the red dots than of the green weakened vection strength because of the lower effective stimulus contrast. In order to test this possibility,

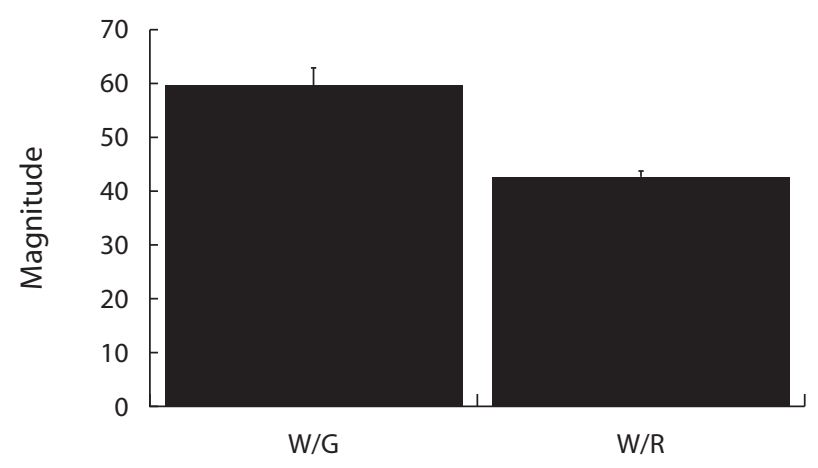

Figure 4. Average magnitude of vection for the 12 subjects in each condition in Experiment 2. The abscissas indicate the colors of the dots and the backgrounds: W/G, white dots on green background; W/R, white dots on red background. The error bars indicate standard errors. 


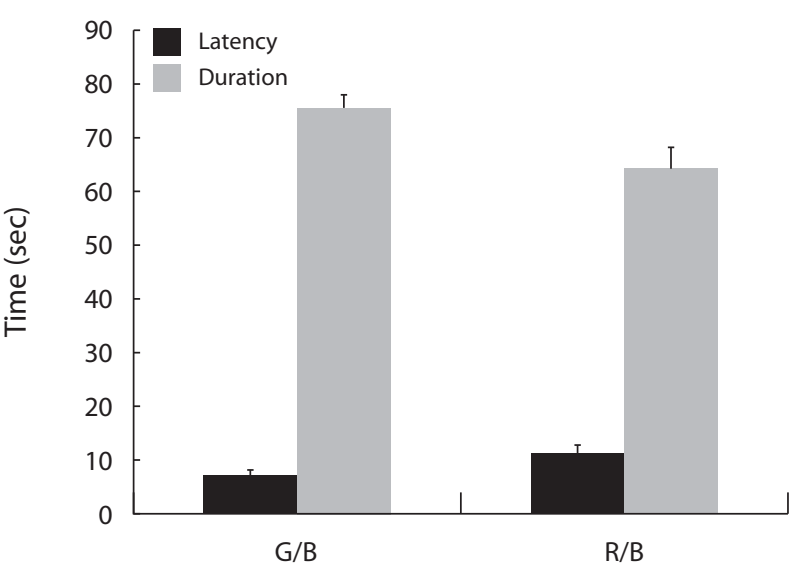

Figure 5. Average latency and duration of vection for the 12 subjects in each condition in Experiment 3. G/B, green dots on black background; $R / B$, red dots on black background. The error bars indicate standard errors.

we employed a background with higher luminance than that of the dots, leaving the dots' luminance unchanged from that in Experiment 3. If the results in Experiment 3 were caused by an artifact in effective luminance settings, stronger vection in the red dot condition could be obtained in this experiment, because a color that possibly had a lower effective luminance in Experiment 3 (i.e., the R/B condition) could give a higher effective luminance contrast with a white background (i.e., the R/W condition).

\section{Method}

The color of the background in the optic flows was changed to white of $8.0 \mathrm{~cd} / \mathrm{m}^{2}$. Thus, the optic flow patterns consisted of two conditions: the green-dots-on-a-white-background (G/W) condition and the red-dots-on-a-white-background $(\mathrm{R} / \mathrm{W})$ condition. The luminances of the red and green dots were the same as those in Experiment 1.

\section{Results and Discussion}

Very weak vection was obtained in the two $(\mathrm{G} / \mathrm{W}$ and $\mathrm{R} / \mathrm{W}$ ) conditions. One of the subjects could not perceive vection at all in either condition. Another subject could perceive vection only in the $\mathrm{G} / \mathrm{W}$ condition. Although vection was weak, all indices of vection showed the same trends as those in Experiment 3. The latency became longer, the duration became shorter, and the magnitude values became smaller in the R/W condition than in the $\mathrm{G} / \mathrm{W}$ condition (Figures 9 and 10). For 2 subjects in the R/W condition, the latency, duration, and magnitude could not be defined, due to lack of a vection response. Thus, we omitted those 2 subjects from data analyses for latency, duration, and magnitude. The $t$ tests revealed significant differences between the two conditions [latency, $t(9)=$ 4.50, $p<.01$; duration, $t(9)=4.06, p<.01$; magnitude, $t(9)=5.43, p<.01]$. These results indicate that vection was weaker in the R/W condition than in the G/W condition. The vection under both conditions in Experiment 5 was much attenuated, possibly because of the low visibility of the dots. The luminance contrast between the dots and the background in Experiment 5 was lower than those in Experiments 1, 2, and 3. By this attenuation, the results became free from the ceiling effect. Thus, the difference between the R/W and G/W conditions was most exaggerated. The possible problem of the difference in dot visibility was addressed later in Experiment 7.

The inhibitory effect of red on vection was observed even though the white background was used. Therefore, the result that the red dots inhibited vection, which was obtained in Experiment 3, could not have been caused by any luminance artifacts. In the next experiment, we further examined the possible effects of luminance artifacts in the previous experiments.

\section{EXPERIMENT 6}

In Experiments 1-5, we applied a flicker photometry method using the entire visual field. However, the measurement method was relatively rough. Thus, there might have been some errors in effective luminance settings. In Experiment 6, we investigated the effect of varied luminance contrasts that could correspond to possible errors in effective luminance settings in the former experiments. We used achromatic dots and backgrounds for this purpose. On the basis of the results, we verified that the results acquired in Experiments 1-5 were not caused by luminance artifacts. These measurements also provided the control conditions for the former experiments. Thus, we compiled the results here and those from Experiments 1-5 in this section.

\section{Method}

The color of the background in the optic flow pattern was varied in four luminances of gray $\left(0.1,1.0,2.1\right.$, and $\left.3.0 \mathrm{~cd} / \mathrm{m}^{2}\right)$, and the colors of the dots were varied as a white of $36.3 \mathrm{~cd} / \mathrm{m}^{2}$, a gray of $4.2 \mathrm{~cd} / \mathrm{m}^{2}$, and a black of $0.0 \mathrm{~cd} / \mathrm{m}^{2}$. Thus, the optic flow patterns consisted of 12 conditions ( 4 dot $\times 3$ background luminances). The stimulus presentation was repeated three times, and the order of the stimulus presentation was randomized.

\section{Results and Discussion}

The results are shown in Figure 11. In the latency, a two-way ANOVA revealed that the main effect of dot luminance was significant $[F(2,22)=11.45, p<.05]$.

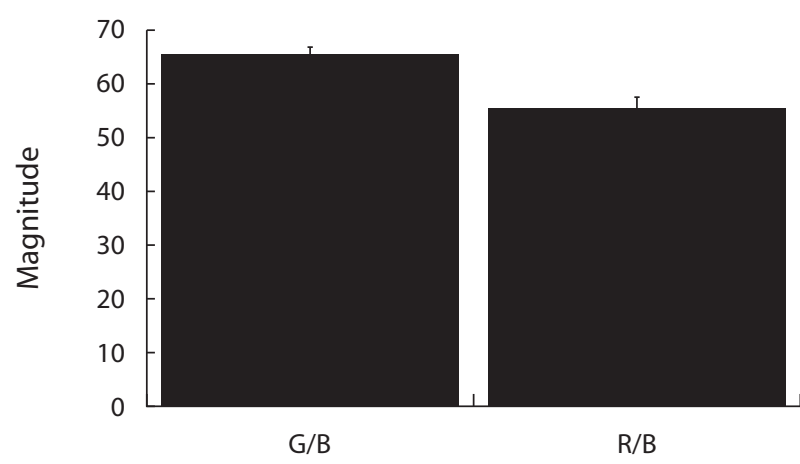

Figure 6. Average magnitude of vection for the 12 subjects in each condition in Experiment 3. The abscissas indicate the colors of the dots: G/B, green dots on black background; R/B, red dots on black background. The error bars indicate standard errors. 


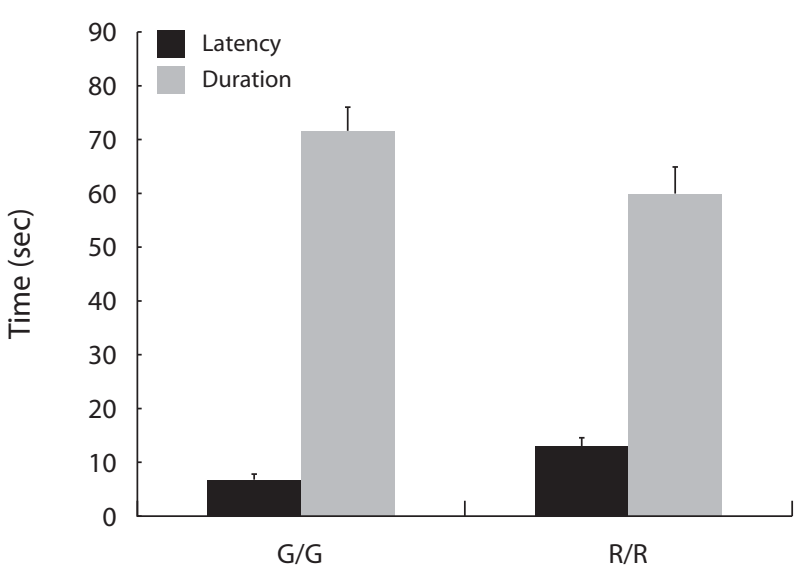

Figure 7. Average latency and duration of vection for the $\mathbf{1 0}$ subjects in each condition in Experiment 4. The abscissas indicate the colors of the dots and backgrounds: $G / G$, green dots on green background; $R / R$, red dots on red background. The error bars indicate standard errors.

There were significant differences between the black dot and gray dot conditions and between the black dot and white dot conditions $(p<.05)$. The main effect of background luminance was not significant $[F(3,33)=0.24$, $p>.05]$. The interaction was not significant $[F(6,66)=$ $0.54, p>.05]$. The luminance differences in the background did not affect the latency of vection if the range was within $0.1-3.0 \mathrm{~cd} / \mathrm{m}^{2}$. On the other hand, dots of $0.0 \mathrm{~cd} / \mathrm{m}^{2}$ induced significantly weaker vection. Drastic luminance contrast change (reversing the polarity) did affect vection strength.

For duration, a two-way ANOVA revealed that the main effect of dot luminance was significant $[F(2,22)=19.43$, $p<.05]$. There were significant differences between the black dot and gray dot conditions and between the black dot and white dot conditions $(p<.05)$. The main effect of background luminance was not significant $[F(3,33)=$ $0.56, p>.05]$. The interaction was also not significant $[F(6,66)=0.32, p>.05]$. These statistics are quite consistent with those from the latency data.

For magnitude, a two-way ANOVA revealed that the main effect of dot luminance was significant $[F(2,22)=$ $36.20, p<.05]$. There were significant differences between the black dot and gray dot conditions and between the black dot and white dot conditions $(p<.05)$. The main effect of background luminance was also significant $[F(3,33)=3.43, p<.05]$. There was a significant difference between the $0.1-$ and $3.0-\mathrm{cd} / \mathrm{m}^{2}$ conditions. The interaction was not significant $[F(6,66)=1.21, p>$ $.05]$. The absolute value of the background may be the critical factor weakening subjective vection strength. If the background luminance is high, vection strength is weakened, and vice versa. However, it is not plausible that there was such a large effective luminance difference (corresponding to a $3-\mathrm{cd} / \mathrm{m}^{2}$ difference) between red and green backgrounds in the former experiments. The red luminance to be matched by green was fixed at $1.0 \mathrm{~cd} / \mathrm{m}^{2}$, whereas individually matched green luminances ranged from 1.0 to $1.2 \mathrm{~cd} / \mathrm{m}^{2}$. Thus, it is difficult for matched green to effectively contain errors in luminance settings corresponding to $3 \mathrm{~cd} / \mathrm{m}^{2}$. On the other hand, the effect of dot luminance was not different between the 4.2- and $36.3-\mathrm{cd} / \mathrm{m}^{2}$ conditions when the background luminance was between 0.1 and $3.0 \mathrm{~cd} / \mathrm{m}^{2}$. The red dot luminance used in the former experiments was $5.6 \mathrm{~cd} / \mathrm{m}^{2}$, whereas the individually matched green luminances were between 5.5 and $5.7 \mathrm{~cd} / \mathrm{m}^{2}$. Thus, it is also implausible that possible luminance-setting artifacts for dots were large enough to weaken vection. Therefore, our results in Experiments 1-5 cannot be explained by luminance artifacts only.

The reversed luminance polarity caused weaker vection in both Experiments 5 and 6. We think that the reversed polarity weakened vection through an illusory depth effect; that is, the brighter background may have been perceived as nearer than the darker dots. The nearer stimulus induces weaker vection (Ohmi, Howard, \& Landolt, 1987). This might contribute to weakening vection. This hypothesis is further discussed in the General Discussion section.

\section{Quantitative Comparisons for Experiments 1-6}

We measured vection in the gray/gray condition (4.2- $\mathrm{cd} / \mathrm{m}^{2}$ dots on a $1.0-\mathrm{cd} / \mathrm{m}^{2}$ background) in Experiment 6 . This could be compared with the R/R and $\mathrm{G} / \mathrm{G}$ conditions in Experiment 4. A one-way ANOVA revealed a main effect of stimulus color [latency, $F(2,22)=4.9$, $p<.05$; duration, $F(2,22)=4.4, p<.05$; magnitude, $F(2,22)=24.1, p<.05]$. Multiple comparisons revealed significant differences between the $\mathrm{R} / \mathrm{R}$ condition and each of the other two conditions in latency, duration, and magnitude $(p<.05)$. Thus, only the $\mathrm{R} / \mathrm{R}$ condition seems to produce weaker vection. Facilitation and inhibition did not occur in the green condition. We also used the $\mathrm{W} /$ gray condition $\left(36.3-\mathrm{cd} / \mathrm{m}^{2}\right.$ dots on a $1.0-\mathrm{cd} / \mathrm{m}^{2}$ background) in Experiment 6 . This could be compared with the W/R and the W/G conditions in Experiment 2. A one-way ANOVA revealed a main effect of background color [latency, $F(2,22)=4.2, p<.05$; duration, $F(2,22)=5.4, p<.05$; magnitude, $F(2,22)=5.7, p<$ $.05]$. Multiple comparisons revealed significant differ-

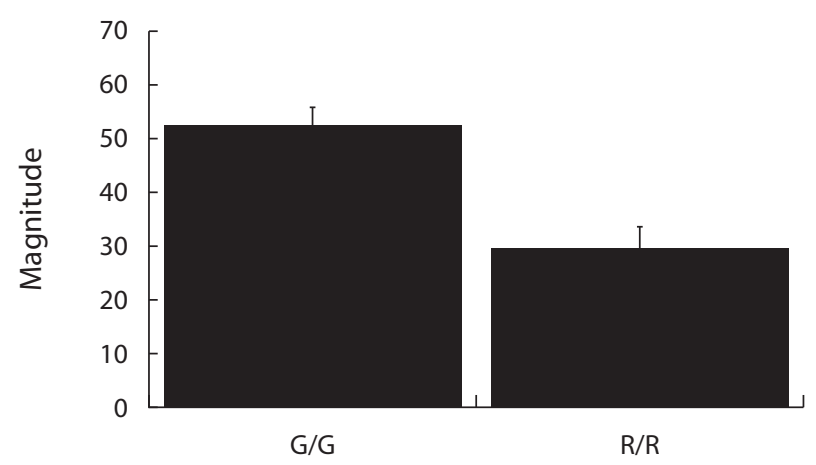

Figure 8. Average magnitude of vection for the 10 subjects in each condition in Experiment 4. The abscissas indicate the colors of the dots and backgrounds: $G / G$, green dots on green background; $R / R$, red dots on red background. The error bars indicate standard errors. 


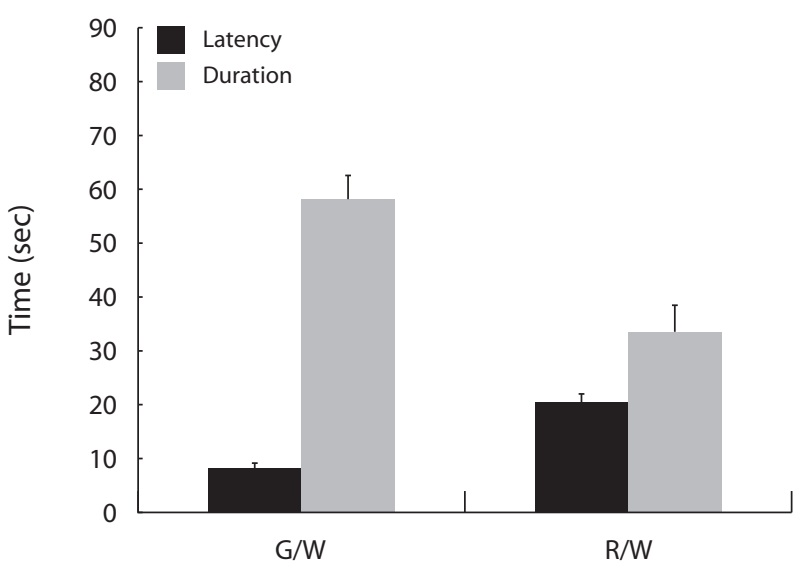

Figure 9. Average latency and duration of vection for the 12 subjects in each condition in Experiment 5. The abscissas indicate the colors of the dots and the backgrounds: G/W, green dots on white background; $\mathbf{R} / \mathbf{W}$, red dots on white background. The error bars indicate standard errors.

ences between the $\mathrm{W} / \mathrm{R}$ condition and each of the other two conditions in latency, duration, and magnitude $(p<$ $.05)$. Thus, the red background condition produced weaker vection, whereas, again, facilitation and inhibition did not occur in the green background condition.

We analyzed the R/G, G/G, W/G, R/R, G/R, and W/R conditions, using a two-way ANOVA. The two factors were dot color (red, green, or white) and background color (green or red). For duration, the main effect of background color was significant $[F(1,11)=8.00, p<.05]$. The main effect of dot color and the interaction were not significant $[F(2,22)=1.25, p>.05$, and $F(2,22)=2.67, p>.05$, respectively]. For latency, the main effect of background color was significant $[F(1,11)=7.06, p<.05]$. The main effect of dot color and the interaction were not significant $[F(2,22)=1.38, p>.05$, and $F(2,22)=0.57, p>.05$, respectively]. For magnitude, the two main effects were significant [background, $F(1,11)=30.53, p<.05$; dot, $F(2,22)=6.30, p<.05]$. The interaction was not significant $[F(2,22)=2.63, p>.05]$.

To analyze the additivity of the dot and background color effects, we conducted a two-way ANOVA for the magnitude values under the $\mathrm{G} / \mathrm{G}, \mathrm{G} / \mathrm{R}, \mathrm{R} / \mathrm{G}$, and R/R conditions. The two factors were dot color (red or green) and background color (red or green). The main effects of dot and background color were significant [dot, $F(1,11)=$ $12.49, p<.05$; background, $F(1,11)=33.41, p<.05]$. The interaction was also significant $[F(1,11)=5.29$, $p<.05]$. Multiple comparisons revealed that there were significant differences between the $\mathrm{R} / \mathrm{R}$ condition and each of the $\mathrm{R} / \mathrm{G}, \mathrm{G} / \mathrm{R}$, and $\mathrm{G} / \mathrm{G}$ conditions $(p<.05)$. No significant effect was found for the other pairs. These statistics indicate that when the dot or background color is green, the red inhibition effect is relatively weak. In other words, when both the dot and background colors are red, the inhibition effect is prominent. Thus, the effects of the red dot and the red background are not simply additive but, rather, synergistic. For duration and latency, only the background color effect was found to be significant.

In general, the effect of the red background was upheld. On the other hand, the effect of the red dot was not always upheld. This may have been related to large data variations in the white dot condition, which possibly masked the differences between the red and green dot conditions. Another reason is that the dot color effect may be relatively weaker than the background color effect.

\section{EXPERIMENT 7}

The results from Experiments 1-5 indicate that red inhibits vection. However, these conditions might include some unexpected effects - namely, differences in perceived speeds, visibilities of dots, and vividness of motion between the red and green conditions. Thus, we examined vection under conditions that made such effects as matched as possible. We used a red or green sinusoidal grating matched by the minimum motion technique (Cavanagh, MacLeod, \& Anstis, 1987) as a vection stimulus. We assumed that the red and green gratings determined by this method would equate the effects of effective contrast, perceived speed, vividness, and visibility. We compared the vection strengths obtained by the red grating and the green grating.

\section{Method}

The vection stimulus was a red or green vertical sinusoidal grating whose luminance was horizontally modulated. In matching the motion strength, the gratings were superimposed and moved in horizontally opposite directions with respect to each other. The size of the grating was a visual angle of $75^{\circ}$ (horizontal) $\times 60^{\circ}$ (vertical) at a viewing distance of $90 \mathrm{~cm}$. The spatial frequency of the grating was 0.3 cycles/deg. We used virtually continuous motion in the minimum motion technique that was essentially the same as that used in $\mathrm{Ca}$ vanagh et al. (1987). Each motion stimulus consisted of 13 images presented sequentially and repeatedly. The gratings shifted approximately $28^{\circ}$ in phase at every refresh. The refresh rate was $75 \mathrm{~Hz}$. The velocity of motion was $19.2 \mathrm{deg} / \mathrm{sec}$ at the center of the screen. The mean luminance and the contrast of the red grating were $1.1 \mathrm{~cd} / \mathrm{m}^{2}$ and $10 \%$ (Michelson contrast), respectively. The mean luminance and the contrast of the green grating depended on the subject, because they were adjusted by each subject. The range of the matched mean

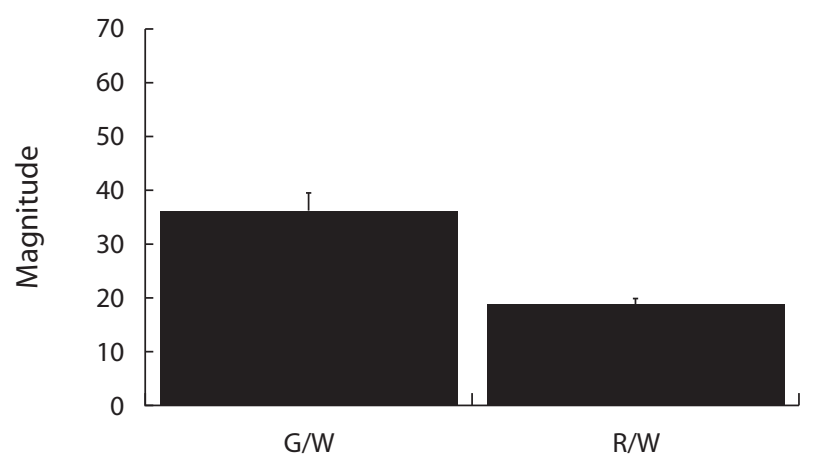

Figure 10. Average magnitude of vection for the 12 subjects in each condition in Experiment 5. The abscissas indicate the colors of the dots and the backgrounds: $G / W$, green dots on white background; $\mathrm{R} / \mathrm{W}$, red dots on white background. The error bars indicate standard errors. 

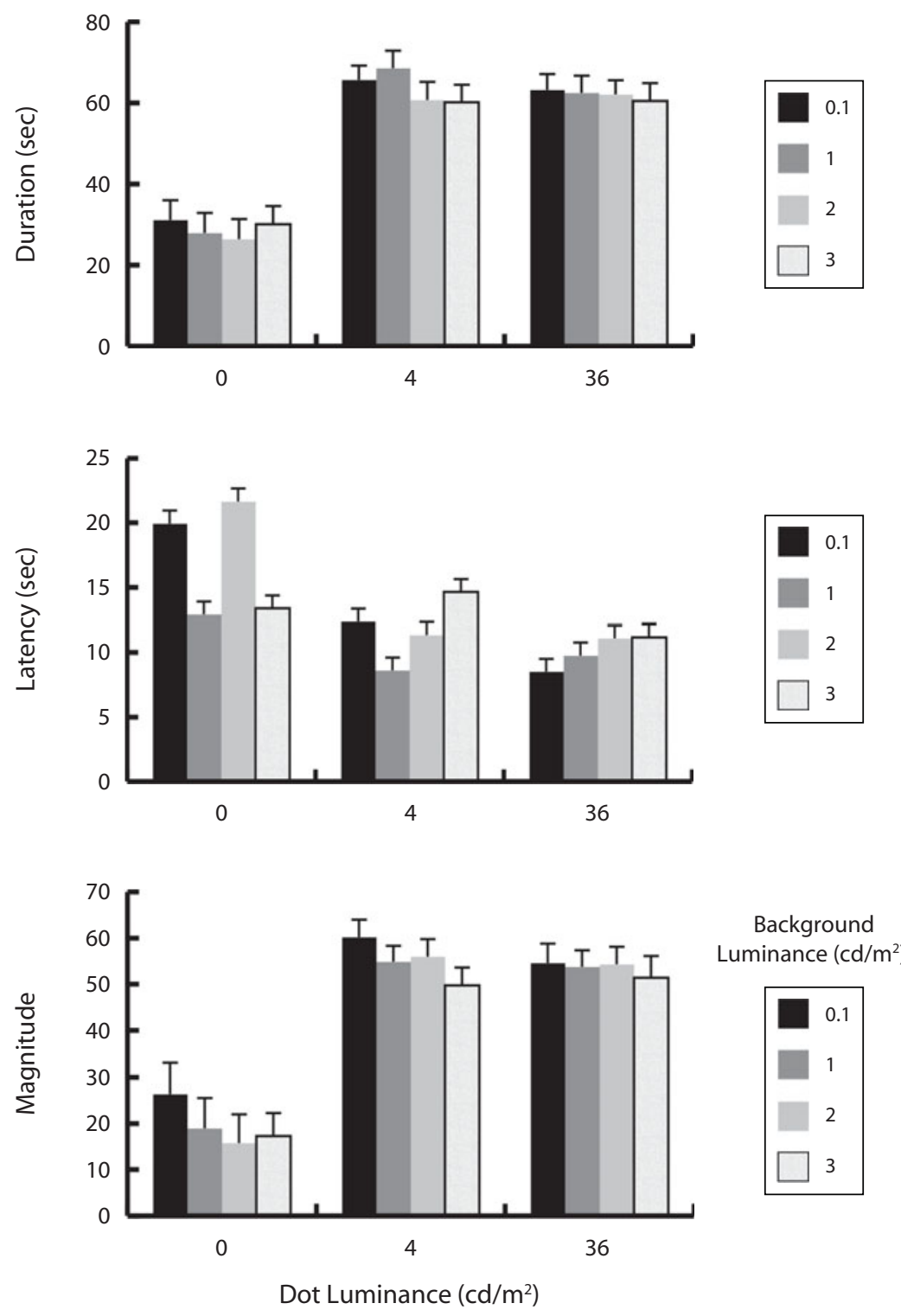

Figure 11. Average duration, latency, and magnitude of vection for the 12 subjects in each condition in Experiment 6. The abscissas indicate the luminances of the dots. The colors of the bars indicate the four background luminances. The error bars indicate standard errors.

luminance of the green grating over 12 subjects was $1.0-1.8 \mathrm{~cd} / \mathrm{m}^{2}$, and its matched contrast over 12 subjects ranged from $7 \%$ to $25 \%$.

The subject adjusted the mean luminance and the contrast of the green by pressing corresponding buttons (increase or decrease the contrast and luminance of the green grating, independently) so that only flicker was seen in the minimum motion stimulus. We measured the latency, the duration, and the subjective strength of vection for the red grating (the red condition) and the green grating (the green condition) in the same way as in the former experiments. The order of the red and green conditions was counterbalanced between the 12 subjects (e.g., half of the 12 subjects performed the red background trials four times successively, followed by the green background trials four times, and the other half performed the trials in the opposite order). Each condition was repeated four times. The grating moved rightward or leftward (the direction was randomized in the four repetitions). Each direction was repeated two times. The duration of the vection stimuli was $100 \mathrm{sec}$. Other procedures were the same as those noted in the General Method section.

\section{Results and Discussion}

Although relatively strong vections were obtained in both conditions, the latency was longer, the duration was shorter, and the subjective strength was weaker in the red condition than in the green condition (Figures 12 and 13). The $t$ tests revealed significant differences between the two conditions [latency, $t(11)=2.95, p<.05$; duration, $t(11)=4.11, p<.01$; magnitude, $t(11)=3.56, p<.01]$, although the amounts of differences in the three indices were small, as compared with those in the results of the former experiments. These results indicate that vection 


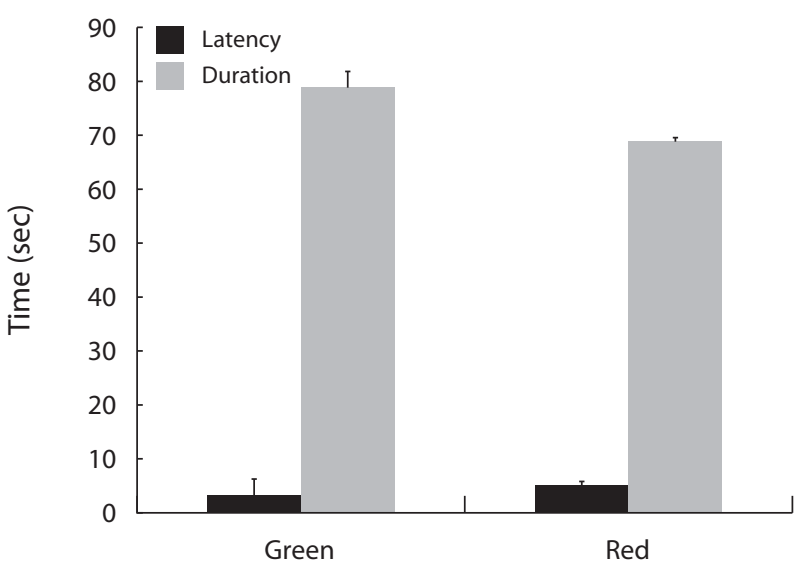

Figure 12. Average latency and duration of vection for the 12 subjects in each condition in Experiment 7. The abscissas indicate the colors of the grating. The error bars indicate standard errors.

was weaker in the red condition than in the green condition even when the grating was used as a stimulus. The two gratings were set to be equal as a whole for the perceived speed, vividness, visibility, and other motion attributes. Thus, inhibition of vection by red cannot be explained by the difference in the visibility of the dots in the former experiments. Red has some inhibitory effects on vection.

\section{GENERAL DISCUSSION}

\section{Vection Is Weakened by Red Visual Stimuli}

In Experiment 1, vection was weaker in the G/R condition than in the $\mathrm{R} / \mathrm{G}$ condition. In Experiment 2, we revealed that the inhibition obtained in Experiment 1 was induced by the red background. We demonstrated that the red dots also have the effect of inhibiting vection (Experiment 3). Furthermore, this inhibition arose from a color effect and not from an interaction between color and luminance, because red inhibited vection even when there was no color difference in the stimulus field (Experiment 4). These results were not caused by a luminance artifact, because the same trends were obtained even when the contrast between the dots and background was reversed (Experiment 5). We also tested the effect of luminance contrasts on vection to examine the possibility of errors in effective luminance settings and found that possible luminance-setting errors could not explain the results in the former experiments (Experiment 6). In addition, vection yielded by a moving grating was also inhibited by red (Experiment 7).

We should remark here that these results do not show that green stimuli simply induce relatively stronger vection than do red stimuli. It is true that the red condition can be interpreted as a lack-of-green condition, resulting in relatively weaker vection. However, vection strength expressed with the values of latency, duration, and magnitude under the $\mathrm{W} / \mathrm{G}$ condition in Experiment 2 was not facilitated, as compared with vection strength under the
W/gray condition that was employed in Experiment 6. On the other hand, those indices show that vection under the $\mathrm{W} / \mathrm{R}$ condition was weaker than that under the W/gray condition. Thus, if the reference color is gray (or white), we should conclude that the green background is not a facilitator or inhibitor, whereas the red background is an inhibitor in vection induction. Furthermore, as was noted earlier, the $\mathrm{R} / \mathrm{R}$ condition induced weaker vection than did each of the $\mathrm{G} / \mathrm{G}$ and gray/gray conditions, whereas the $\mathrm{G} / \mathrm{G}$ condition did not induce stronger vection than the gray/gray condition. This confirms the above-noted red inhibition conclusion.

Red was found to have an inhibitory effect on vection strength when it was employed as the background or as the moving dots. Parts of the present results might be explained in terms of a general motion perception paradigm. It is known that adding chromatic modulation to a moving luminance grating slows down its perceived velocity, in spite of the fact that information about motion can be acquired from both chromatic and achromatic pathways (Cavanagh, Boeglin, \& Favreau, 1985). However, our results also show that the combination of red dots and a red background, not including chromatic motion, was most effective in inhibiting vection. Therefore, it was not adding chromatic motion information but, rather, the red color itself that weakened vection.

\section{Physiological Bases}

Visual pathways are of two types: magnocellular and parvocellular (Shapley, 1990). Magno cells are highly sensitive to transient stimuli and contribute to motion perception (De Yoe \& Van Essen, 1988; Lennie, Trevarthen, Van Essen, \& Wassle, 1990; Schiller, 1986). A subset of magno cells is inhibited selectively by light with long wavelengths - that is, a red light (de Monasterio \& Schein, 1980; Dreher, Fukada, \& Rodieck, 1976; Krüger, 1977; Lee, 1996; Livingstone \& Hubel, 1987; Shapley, 1990; Wiesel \& Hubel, 1966). These magno cells are called Type IV neurons.

These Type IV magno cells may contribute to vection induction. In that case, inhibition of Type IV magno cells can be directly connected with the inhibition of vection. The results of the present study can be explained by as-

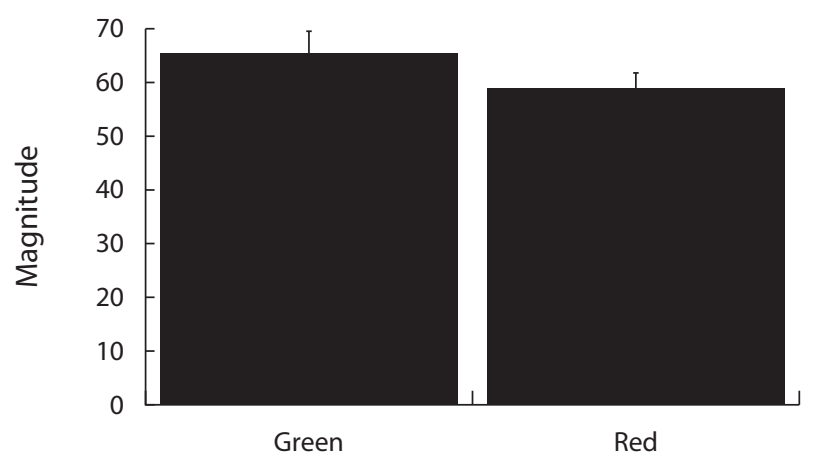

Figure 13. Average magnitude of vection for the 12 subjects in each condition in Experiment 7. The abscissas indicate the colors of the grating. The error bars indicate standard errors. 
suming that the magno cells in the visual pathway are inhibited by red, resulting in vection inhibition.

However, recent studies have revealed not only that a red background cannot isolate magnocellular responses, but also that it affects parvocellular responses (Skottun, 2004). In addition to this, there is a problem with the explanation for the inhibition of vection by subcortical magno cells because the small size of the receptive fields and the lack of direction selectivities of magno cells are not suitable for the processing of dot motion (Skottun \& Skoyles, 2006).

Furthermore, in Experiment 7, although we equated the perceived motion strength, vection strength was weaker in the red condition. If the Type IV magno cells are responsible for general motion signals, the dissociation of motion perception and vection cannot be explained by inhibition of the magno cell activities.

Consequently, we do not propose a hypothesis of magno cell inhibition by red lights for explaining vection inhibition by red, as found here. However, at the same time, we do not deny the possibility that the magnocellular pathway, which is extended to layer 4B of V1 and to higher cortical areas such as MT and MST, may be inhibited selectively for vection by red lights at some stage of processing.

\section{On the Dot Color Effect}

We should explain the effect of red dots that inhibited vection. We propose two possible explanations. One is illusory depth perception of red dots. When red and green targets are presented as stimuli on the same black background, the red target is perceived as nearer than the green one by the color-depth illusion made by ocular chromatic aberration (Winn, Bradley, Strang, McGraw, \& Thibos, 1995). This fact was further supported by a grasping study. The maximal finger aperture decreased more when a red target was employed than when a green target was employed. The red target was perceived as nearer and also misperceived as smaller than the green target (Gentilucci, Benuzzi, Bartolani, \& Gangitano, 2001). Depth is a mediating factor of vection strength, and the farthest stimulus dominates vection direction (e.g., Ito \& Shibata, 2005). It is possible that red targets (red dots) were perceived as nearer than the green targets; thus, vection was attenuated more in red dot conditions than in green ones.

The other explanation for the red dot effect is that sequential retinal stimulation by moving red dots over the screen effectively functioned as the illumination of a red light. In this case, the red dots should have the same effect in quality (but in a smaller amount) as that of the red background. However, it is not clear that the red dots presented here were a strong enough stimulus to function as a red light.

\section{Do Rod Signals Weaken Vection?}

We conducted Experiments 1-7 with the stimulus in relatively low luminance because of the restriction of the apparatus. Therefore, we should consider the possibility of the contribution of rods.

Rod signals have phase delays, relative to cone signals. It is known that there are two types of rod pathways (Stockman \& Sharpe, 2006). One is the fast pathway, whose delay is approximately $33 \mathrm{msec}$, and the other is the slow pathway, whose delay is $67 \mathrm{msec}$. Therefore, to a greater or lesser extent, rod signals would affect motion perception and/or vection in our experimental conditions.

There are a few studies in motion perception with scotopic vision. Gegenfurtner, Mayser, and Sharpe (2000) revealed that the motion detection threshold did not change between photopic and scotopic vision, whereas the perceptual speed of the moving stimulus decreased approximately $20 \%$ with scotopic vision, as compared with photopic vision, if the temporal frequency of the stimulus was below $4 \mathrm{~Hz}$.

In this study, with adaptation to our stimulus, the subjects were considered to be in mesopic vision. Thus, rods may have contributed to effective luminance settings. However, the photopic luminances of green that equated to red were near the photopic luminances of red, whereas the scotopic luminances of the green and the red (backgrounds) were very different - that is, 2.35 and 0.261 scotopic $\mathrm{cd} / \mathrm{m}^{2}$, respectively - when the photopic luminance of the green and red backgrounds was $1.0 \mathrm{~cd} / \mathrm{m}^{2}$. Therefore, settings of the green luminances by our subjects were not executed in near scotopic vision. Although we cannot specify how large the rod contribution is to red and green luminance settings, the possible rod contribution is definitely greater for green, because rods have very low sensitivity to red. When the perceived speed becomes slower as the rod response becomes larger, weakened vection could be obtained for the green motion stimulus, which would appear to be slower than the red motion stimulus. Applying this idea to our stimuli, weakened vection would be observed in the $\mathrm{G} / \mathrm{R}, \mathrm{G} / \mathrm{B}, \mathrm{G} / \mathrm{W}, \mathrm{G} / \mathrm{G}$, and green grating conditions. However, in fact, vection observed in the $R / G, R / B, R / W$, $\mathrm{R} / \mathrm{R}$, and red grating conditions was weakened. Only the G/R and G/W conditions, which, remarkably, weakened vection, were consistent with the expectation above. The results of Experiment 7 were also contrary to the expectation; that is, vection yielded by the moving red grating was weaker than that yielded by the green grating. Moreover, we used 5.8-Hz motion stimuli in Experiment 7, which is sufficiently faster than $4 \mathrm{~Hz}$ (Gegenfurtner et al., 2000). In addition, Grossman and Blake (1999) revealed that there was no difference in the coherent motion threshold between photopic and scotopic vision. Thus, it is difficult to explain our results by the contribution of rods and the attenuation of the perceived speed.

Since visual information processing in scotopic vision is not yet well known, we cannot reject the possibility that there are unknown effects of the rods on vection and/or motion perception. To eliminate the contribution of rods, we should perform the same experiments with red and green backgrounds above the radiance level at which the rod is saturated, such as above $10 \mathrm{~cd} / \mathrm{m}^{2}$. However, at present, it is very difficult to construct such experimental equipment with a large screen. This is a future task.

\section{Related Studies on the Effects of Color on the Self-Motion Perception}

Bonato and Bubka (2006) revealed that stripes consisting of six different colors, including black and white, 
facilitate vection, rather than black and white stripes. Their results suggested that a multicolored stimulus induced stronger vection than did a monocolored stimulus. Thus, it is possible that bicolored stimuli (e.g., R/G, G/R) induced stronger vection than did monocolored stimuli $(\mathrm{R} / \mathrm{R})$. The strongest inhibition under the $\mathrm{R} / \mathrm{R}$ condition may be explained partly by this factor. However, there was an essential difference between Bonato and Bubka's study and ours. There were more than two colored motions in their stimuli; however, there was always only one colored motion in our stimuli. Bonato and Bubka speculated that the complexity of a stimulus is a critical factor in facilitating vection. In our stimuli, the complexity of the moving stimulus (i.e., dots) was not different between mono- and bicolored conditions. We examined the effects of colors - that is, red and green - on vection without increasing the complexity of the stimulus. Thus, we do not think that the effect of Bonato and Bubka played an important role in our study.

Matsuzaki, Kawaguchi, and Kitazaki (2009) used an optic flow with isoluminant red and green backgrounds and measured the accuracy of heading. They reported that the accuracy of heading was higher with a red background than with an isoluminant green background. Even though vection and heading perceptions are different phenomena and their detailed relationship is not adequately known, both are related to self-motion and, possibly, share the same neural mechanisms. It is possible that future research will relate heading and vection paradigms to the effects of colors.

\section{Conclusion}

The effect of a red light or background has been repeatedly demonstrated by psychophysical experiments (e.g., Chapman et al., 2004). This article adds yet another example of inhibition-by-red phenomena. Previous researchers concluded that a red light or background inhibited activity of the magnocellular pathway, which resulted in inhibition in perceptual phenomena. Although we are cautious about the conclusion, as was noted above, we believe that previous red effects and our vection inhibition effect share some common underlying mechanisms. Magnocellular inhibition cannot be totally denied, but a more sophisticated explanation is required. To clarify that mechanism, further physiological studies are required.

\section{AUTHOR NOTE}

This study was partly funded by a Grant-in-Aid for Scientific Research $(20300048,19103003,21830081)$ provided by the Ministry of Education, Culture, Sports, Science, and Technology-Japan, and also by a grant from the Nissan Science Foundation. Correspondence concerning this article should be addressed to T. Seno, Faculty of Design, Kyushu University, 4-9-1 Shiobaru, Minami-ku, Fukuoka 815-8540, Japan (e-mail: seno@design.kyushu-u.ac.jp).

\section{REFERENCES}

Bedwell, J. S., Brown, J. M., \& Miller, L. S. (2003). The magnocellular visual system and schizophrenia: What can the color red tell us? Schizophrenia Research, 63, 273-284.

Bonato, F., \& BubKa, A. (2006). Chromaticity, spatial complexity, and self-motion perception. Perception, 35, 53-64.
Brandt, T., Dichgans, J., \& Koenig, E. (1973). Differential effects of central versus peripheral vision on egocentric and exocentric motion perception. Experimental Brain Research, 16, 476-491.

Breitmeyer, B. G., \& Williams, M. C. (1990). Effects of isoluminantbackground color on metacontrast and stroboscopic motion: Interactions between sustained $(P)$ and transient $(M)$ channels. Vision Research, 30, 1069-1075.

Bubka, A., Bonato, F., \& Palmisano, S. (2008). Expanding and contracting optic-flow patterns and vection. Perception, 37, 704-711.

Cavanagh, P., Boeglin, J., \& Favreau, O. E. (1985). Perception of motion in equiluminous kinematograms. Perception, 14, 151-162.

Cavanagh, P., MacLeod, D. I. A., \& Anstis, S. M. (1987). Equiluminance: Spatial and temporal factors and the contribution of blue-sensitive cones. Journal of the Optical Society of America A, 4, 1428-1438.

Chapman, C., Hoag, R., \& Giaschi, D. (2004). The effect of disrupting the human magnocellular pathway on global motion perception. $V i$ sion Research, 44, 2551-2557.

Chase, C., Ashourzadeh, A., Kelly, C., Monfette, S., \& Kinsey, K. (2003). Can the magnocellular pathway read? Evidence from studies of color. Vision Research, 43, 1211-1222.

De Monasterio, F. M., \& Schein, S. J. (1980). Protan-like spectral sensitivity of foveal Y ganglion cells of the retina of macaque monkeys Journal of Physiology, 299, 385-396.

De Yoe, E. A., \& VAN EsSEn, D. C. (1988). Concurrent processing streams in monkey visual cortex. Trends in Neurosciences, 11, 219-226.

Dreher, B., Fukada, Y., \& Rodieck, R. W. (1976). Identification, classification and anatomical segregation of cells with X-like and Y-like properties in the lateral geniculate nucleus of old-world primates. Journal of Physiology, 258, 433-452.

Edwards, M., BADCOCK, D. R., \& Nishida, S. (1996). Contrast sensitivity of the motion system. Vision Research, 36, 2411-2421.

Fischer, M. H., \& KorNMUller, A. E. (1930). Optokinetisch ausgelöste Bewegungswahrnehmung und optokinetischer Nystagmus. Journal of Psychological Neurology, 41, 273-308.

Gegenfurtner, K. R., Mayser, H. M., \& Sharpe, L. T. (2000). Motion perception at scotopic light levels. Journal of the Optical Society of America A, 17, 1505-1515.

Gentilucci, M., Benuzzi, F., Bartolani, L., \& Gangitano, M. (2001). Influence of stimulus color on the control of reachinggrasping movements. Experimental Brain Research, 137, 36-44.

Grossman, E. D., \& BlaKe, R. (1999). Perception of coherent motion, biological motion and form-from-motion under dim-light conditions. Vision Research, 39, 3721-3727.

Iто, H., \& Shiвata, I. (2005). Self-motion perception from expanding and contracting optical flows overlapped with binocular disparity. $\mathrm{Vi}$ sion Research, 45, 397-402.

KITAZAKI, M., \& SATO, T. (2003). Attentional modulation of self-motion perception. Perception, 32, 475-484.

KRÜGER, J. (1977). Stimulus dependent colour specificity of monkey lateral geniculate neurones. Experimental Brain Research, 30, 297-311.

LEE, B. B. (1996). Receptive field structure in the primate retina. Vision Research, 36, 631-644.

Lennie, P., Trevarthen, C., Van Essen, D., \& Wassle, H. (1990). Parallel processing of visual information. In L. Spillman \& J. S. Werner (Eds.), Visual perception: The neurological foundations (pp. 103128). San Diego: Academic Press.

LepecQ, J.-C., Giannopul, I., \& Baudonniere, P.-M. (1995). Cognitive effects on visually induced body motion in children. Perception, 24, 435-449.

Livingstone, M. S., \& Hubel, D. H. (1987). Psychophysical evidence for separate channels for the perception of form, color, movement, and depth. Journal of Neuroscience, 7, 3416-3468.

Matsuzaki, N., Kawaguchi, K., \& Kitazaki, M. (2009). Contribution of parvocellular pathway in heading perception: Effects of background color, element size, and eccentricity [In Japanese with English abstract]. Vision, 21, 1-11.

Michimata, C., OKubo, M., \& Mugishima, Y. (1999). Effects of background color on the global and local processing of hierarchically organized stimuli. Journal of Cognitive Neuroscience, 11, 1-8.

Ohmi, M., Howard, I. P., \& Landolt, J. P. (1987). Circular vection as a function of foreground-background relationships. Perception, 16, 17-22.

Palmisano, S., \& Chan, A. Y. C. (2004). Jitter and size effects on vec- 
tion are immune to experimental instructions and demands. Perception, 33, 987-1000.

Pammer, K., \& Lovegrove, W. (2001). The influence of color on transient system activity: Implications for dyslexia research. Perception \& Psychophysics, 63, 490-500.

Schiller, P. H. (1986). The central visual system. Vision Research, 26, $1351-1386$

Seno, T., \& Sato, T. (2009). Positional and directional preponderances in vection. Experimental Brain Research, 192, 221-229.

Shapley, R. (1990). Visual sensitivity and parallel retinocortical channels. Annual Review of Psychology, 41, 635-658.

Skottun, B. C. (2004). On the use of red stimuli to isolate magnocellular responses in psychophysical experiments: A perspective. Visual Neuroscience, 21, 63-68.
Skottun, B. C., \& Skoyles, J. R. (2006). Is coherent motion an appropriate test for magnocellular sensitivity? Brain \& Cognition, 61, 172-180.

Stockman, A., \& Sharpe, L. T. (2006). Into the twilight zone: The complexities of mesopic vision and luminous efficiency. Ophthalmic \& Physiological Optics, 26, 225-239.

Wiesel, T. N., \& Hubel, D. H. (1966). Spatial and chromatic interactions in the lateral geniculate body of the rhesus monkey. Journal of Neurophysiology, 29, 1115-1156.

Winn, B., Bradley, A., Strang, N. C., McGraw, P. V., \& Thibos, L. N. (1995). Reversals of the colour-depth illusion explained by ocular chromatic aberration. Vision Research, 35, 2675-2684.

(Manuscript received August 11, 2009; revision accepted for publication March 30, 2010.) 